\title{
Novel real-time cell analysis platform for the dynamic monitoring of ionizing radiation effects on human tumor cell lines and primary fibroblasts
}

\author{
IMOLA MÁN ${ }^{1}$, GÁBOR J. SZEBENI ${ }^{1}$, IMOLA PLANGÁR ${ }^{2}$, EMILIA R. SZABÓ ${ }^{3}$, TÜNDE TŐKÉS ${ }^{4}$, \\ ZOLTÁN SZABÓ ${ }^{3}$, ZOLTÁN NAGY ${ }^{3}$, GÁBOR FEKETE ${ }^{3}$, ROBERTA FAJKA-BOJA ${ }^{5}$, \\ LÁSZLÓ G. PUSKÁS ${ }^{1}$, KATALIN HIDEGHÉTY ${ }^{3}$ and LÁSZLÓ HACKLER Jr. ${ }^{1}$
}

\author{
${ }^{1}$ Avidin Ltd., Szeged H-6726; ${ }^{2}$ Department of Neurology, Faculty of Medicine, University of Szeged, Szeged H-6725; \\ ${ }^{3}$ Department of Oncotherapy; ${ }^{4}$ Institute of Surgical Research, Faculty of Medicine, University of Szeged, Szeged H-6720; \\ ${ }^{5}$ Institute of Genetics, Biological Research Centre, Hungarian Academy of Sciences, Szeged H-6726, Hungary
}

Received September 29, 2014; Accepted May 28, 2015

DOI: $10.3892 / \mathrm{mmr} .2015 .4004$

\begin{abstract}
Translational research in radiation oncology is important for the detection of adverse radiation effects, cellular responses, and radiation modifications, and may help to improve the outcome of radiation therapy in patients with cancer. The present study aimed to optimize and validate a real-time label-free assay for the dynamic monitoring of cellular responses to ionizing radiation. The xCELLigence system is an impedance-based platform that provides continuous information on alterations in cell size, shape, adhesion, proliferation, and survival. In the present study, various malignant human primary fibroblast cells (U251, GBM2, MCF7, A549, HT-29) were exposed to 0,5 and 10 Gy of Cobalt ${ }^{60}$ radiation. As well as the xCELLigence system, cell survival and proliferation was evaluated using the following conventional end-point cell-based methods: Clonogenic, MTS, and lactate dehydrogenase assays, and apoptosis was detected by fluorescence-activated cell sorting. The effects of ionizing radiation were detected for each cell line using impedance monitoring. The real-time data correlated with the colony forming assay results. At low cell densities (1,000-2,000 cells/well) the impedance-based method was more accurate at monitoring dose-dependent changes in the malignant human primary fibroblast cell lines, as compared with the end-point assays. The results of the present study demonstrated that the xCELLigence system may be a reliable and rapid diagnostic method for the monitoring of dynamic cell behavior following radiation. In addition, the $\mathrm{xCELLigence} \mathrm{system} \mathrm{may} \mathrm{be} \mathrm{used} \mathrm{to}$ investigate the cellular mechanisms underlying the radiation
\end{abstract}

Correspondence to: Dr László Hackler Jr., Avidin Ltd., Alsó Kikötő sor 11, Szeged H-6726, Hungary

E-mail: hackler@avidinbiotech.com

Key words: ionizing radiation, cell viability, in vitro assays, $\mathrm{xCELLigence}$ response, as well as the time-dependent effects of radiation on cell proliferation and viability.

\section{Introduction}

Translational research in radiation oncology is important for the detection of adverse radiation effects, cellular responses, and radiation modifications, and may help to improve the outcome of radiation therapy in patients with cancer (1). The simultaneous administration of chemotherapy and radiation therapy may enhance tumor cell destruction, and protective agents [e.g. Prussian blue, calcium diethylenetriamene pentaacetate (DTPA) and zinc DTPA, potassium iodide and amifostine] (2) may reduce acute inflammatory reactions and healthy tissue complications (3). Further studies on the optimization of the dosage and timing of the components of such a binary therapeutic system (radiation and radiation modifiers) are crucial to the development of novel clinical therapeutic strategies (4). Therefore, there is a requirement for novel in vitro models that better reflect in vivo conditions, which may be reliably transferred into clinical settings. To date, limited information is available regarding changes in cellular proliferation and viability over time, due to the cytotoxicity of certain therapeutic agents, or to ionizing radiation. Novel real-time cell analysis platforms allow the continuous detection of cell viability during a defined period following anti-cancer treatment (5).

Clonogenic, MTS, and lactate dehydrogenase (LDH) assays are well-known end-point methods used for the evaluation of chemoradiation and radiosensitivity.

The clonogenic assay is commonly used to investigate the survival and proliferative capacity of irradiated cancer cells. The clonogenic assay is used to measure the ability of cells to grow in an anchorage-dependent manner. If the cells are able to proliferate, they grow in cellular aggregates, referred to as colonies. The limiting factor for the use of this method is the length of time taken for a colony to form, which may take days or weeks. The colonies are then quantified following crystal violet staining (6). 
The MTS assay is used to examine the chemosensitivity or toxicity of drugs in human tumor cell lines, and to quantify the survival of cancer cells following radiation (7). The MTS assay is composed of a cell-permeable, soluble tetrazolium salt, and an electron coupling reagent (phenazine methosulfate) that are added to the cellular environment following appropriate dosing periods. During incubation with MTS, the dehydrogenase enzymes present within the mitochondria of healthy cells reduce the MTS to a dark purple, water-soluble formazan compound, which may then be quantified $(8,9)$.

Another method of detecting cytotoxicity is the assessment of membrane integrity, which is achieved by monitoring the passage of substances that are normally sequestered inside cells to the outside of the cells. During the LDH assay, the release of $\mathrm{LDH}$ from the cytoplasm into the supernatant indicates cell lysis (10).

Previous studies have used cell microelectronic sensing to assess the real-time cytoprotective effects of novel drug candidates (11), to study the toxicity of various compounds in brain endothelial and epithelial cells (12), and to dynamically monitor the integrity of experimental biological barriers (13-15).

The xCELLigence system, which was co-developed by Roche (Budapest, Hungary) and ACEA Biosciences, Inc., (San Diego, CA, USA) provides real-time continuous monitoring and label-free assessment of cellular adhesion, morphological changes, proliferation, viability, and cytotoxicity, thereby revealing the physiological state of cells. The system consists of four main components: A Real-Time Cell Analyzer (RTCA), an RTCA Double Plate station, an RTCA computer with integrated software, and microtiter plates (E-plate 16). The core of the system is composed of disposable microelectronic cell sensor arrays integrated into the bottom of the E-plates, which perform cell-based assays on the RTCA instrument. The size of the E-plate 16 corresponds to $1 / 6$ of the size of a 96 -well microtiter plate, with 16 wells contained in two side by side columns $(16,17)$. The electronic impedance of the electrode sensors is measured in order to allow the monitoring and detection of any alterations in cellular attachment $(18,19)$. The impedance measured between the electrodes in an individual well depends on electrode geometry, the ionic concentration of the well, and whether the cells are attached to the electrodes. In the absence of cells, electrode impedance is predominantly determined by the ionic environment, both at the electrode/solution interface, and in the bulk solution. In the presence of cells, the cells attached to the electrode sensor surface act as insulators, thereby altering the local ionic environment at the electrode/solution interface, leading to an increase in impedance. The more cells that have grown on the electrodes, the larger the measured electrode impedance. The impedance measurement, which is displayed as a cell index $(\mathrm{CI})$, provides quantitative information regarding the biological status of the cells, including cell number, viability, and morphology. Calculation of the cell index is based on the following formula: $\mathrm{CI}=\left(\mathrm{Z}_{\mathrm{i}}-\mathrm{Z}_{0}\right) / 15$, where $\mathrm{Z}_{\mathrm{i}}$ is the impedance at an individual time point during the experiment, and $\mathrm{Z}_{0}$ is the impedance at the start of the experiment. Therefore, the $\mathrm{CI}$ is a self-calibrated value derived from the ratio of impedance. Impedance-based monitoring of cell proliferation and viability correlates accurately with cell number $(20,21)$. The present study used a 16 -well plate system that is able to simultaneously measure three independent 16 -well plates. This allowed the simultaneous investigation of two doses of irradiation, together with non-irradiated control cells.

The present study presents the advantages and the potential uses of the novel xCELLigence biosensor system for the measurement of the effects of ionizing radiation on tumor and primary cells, as compared with alternative cell-based assays.

\section{Materials and methods}

Cell lines and culture conditions. U251 human glioblastoma, A549 human lung carcinoma, MCF7 human breast adenocarcinoma, HT-29 human colon adenocarcinoma and human primary fibroblast cells (passage 4) were all purchased from the ATCC (Wesel, Germany), and stored at $-80^{\circ} \mathrm{C}$ in a solution containing $90 \%$ fetal bovine serum (FBS; Gibco Life Technologies, Budapest, Hungary) and 10\% dimethyl sulfoxide (DMSO; Molar Chemicals Kft., Budapest, Hungary). GBM2 human glioblastoma cells were obtained from Dr Balázs Hegedűs (National Oncology Institute, Budapest, Hungary). The human primary fibroblast cells were cultured in low glucose Dulbecco's modified Eagle's medium (DMEM; Lonza, Budapest, Hungary); the A549, MCF7, HT-29, and GBM2 cells were cultured in DMEM-F12 (Lonza); and the U251 cells were cultured in RPMI-1640 medium (Lonza) supplemented with 10\% heat-inactivated FBS (Gibco Life Technologies), $1 \%$ L-glutamine, $100 \mathrm{U} / \mathrm{ml}$ penicillin, and $100 \mu \mathrm{g} / \mathrm{ml}$ streptomycin (Sigma-Aldrich, Budapest, Hungary) at $37^{\circ} \mathrm{C}$ in a humidified atmosphere containing $5 \% \mathrm{CO}_{2}$.

Irradiation. A Teragam K-01 cobalt unit (SKODA-ÚJP, Prague, Czech Republic) was used (average energy $1.25 \mathrm{MeV}$; source/image distance, $80 \mathrm{~cm}$ ) to irradiate the cells seeded into 16-well E-plates (Roche), and 96-well and 6-well normal tissue culture plates. The plates were surrounded by water on either side and placed between two $2 \mathrm{~cm}$ polymethyl methacrylate slabs to ensure adequate material build-up. The isocentre was positioned at the geometrical centers of the plates. Half of the radiation dose was delivered with a downward 20x20 cm beam (gantry angle, $0^{\circ}$ ), whereas the other half of the radiation dose was delivered with an upward $20 \times 20 \mathrm{~cm}$ beam (gantry angle, $180^{\circ}$ ), in order to maximize field homogeneity. The delivered doses were 0, 5, and $10 \mathrm{~Gy}$. Due to the decay of Cobalt $^{60}$, irradiation time correction factors were applied.

Clonogenic survival assay. For irradiation experiments, the cells were grown in $75 \mathrm{~cm}^{2}$ flasks, until they reached $75 \%$ confluence. The cells were then harvested by trypsinization (Sigma-Aldrich Chemie GmbH, Schnelldorf, Germany), and counted using Bürker chambers. The cells were subsequently seeded at 1,000 cells/well into 6-well plates, and allowed to adhere for $24 \mathrm{~h}$. All clonogenic assay experiments were performed in triplicate. During 8 days, the irradiation medium was changed every 2 days. The medium was then discarded and the cells were washed with phosphate-buffered saline (PBS) prior to fixation with $4 \%$ paraformaldehyde. Colony forming units (CFUs) were stained with a solution containing $0.5 \%$ crystal violet (Sigma-Aldrich Chemie 
$\mathrm{GmbH}$ ) for $20 \mathrm{~min}$. The plates were rinsed three times with tap water and left to dry at room temperature. Colony counting was conducted the following day using the Zeiss Axiovert 25 microscope (Zeiss, Oberkochen, Germany), with the number of CFUs expressed as a percentage, as compared with the control samples, which were considered $100 \%$.

MTS cell viability assay. The viability of each cell line was determined using a colorimetric MTS assay. The cells were seeded into 96-well tissue culture plates at a density of 500 , $1,000,2,000$, or 4,000 cells/well, and cultured overnight prior to irradiation. Three days following irradiation, $20 \mu \mathrm{l}$ MTS (Gibco Life Technologies) was added at a working concentration of $0.3 \mathrm{mg} / \mathrm{ml}$, and incubated for $\geq 1 \mathrm{~h}$ at $37^{\circ} \mathrm{C}$, in an atmosphere containing $5 \% \mathrm{CO}_{2}$. Following incubation, the absorbance was measured using a Wallac 1420 VICTOR2 $2^{\mathrm{TM}}$ ELISA assay plate reader (Wallac VICTOR Plate Reader; PerkinElmer, Inc., Waltham, MA, USA) at a wavelength of $490 \mathrm{~nm}$, with the values expressed in arbitrary units. All MTS cell viability experiments were performed in triplicate.

Lactate dehydrogenase ( $L D H)$ assay. A Cytotoxicity Detection kit (Roche) was used to assess cell viability following irradiation. An LDH assay measures the activity levels of $\mathrm{LDH}$ in the supernatant released from damaged cells, or the activity levels of total LDH following cell lysis. The former correlates with the number of dead cells, whereas the latter correlates with the number of viable cells. For the present study, given the low cell numbers, the release assay did not provide sufficient information, likely due to the relatively low levels of cell apoptosis, as well as the low sensitivity of the assay. Therefore, the total LDH activity level detection method was used following cell lysis. The cells were seeded at four densities: 500, 1,000, 2,000, and 4,000 cells/well. Three days following irradiation, $70 \mu \mathrm{l}$ supernatant was removed from the wells and plated into a 96-well plate. The remaining medium was discarded. The cells were then washed with PBS, and $70 \mu \mathrm{l}$ PBS containing 1\% Triton X-100 (Sigma-Aldrich Chemie GmbH) was added to the cells to achieve total cell lysis. Following $15 \mathrm{~min}$ incubation at room temperature, $70 \mu \mathrm{LDH}$ reagent was added to both the lysed cells and the wells containing the supernatant, and absorbance at $490 \mathrm{~nm}$ was recorded at $2 \mathrm{~min}$ intervals for a duration of $20 \mathrm{~min}$.

Cell growth and proliferation assay using xCELLigence. The cells were grown in $100 \mathrm{~mm}$ Petri dishes until they had reached $90 \%$ confluence; the cells were subsequently harvested by trypsinization, and seeded into 16-well E-plates at various densities in $100 \mathrm{ml}$ medium $(500,1,000,2,000$, 4,000 cells/well). Following seeding, the cells were monitored every $10 \mathrm{~min}$ by the xCELLigence system (Roche) for proliferation, attachment, and spreading. Following $24 \mathrm{~h}$, during which time the cells entered the exponential growth phase, the cells were irradiated with 5 and 10 Gy doses, and impedance detection via the incorporated sensor electrode arrays continued for a further $76 \mathrm{~h}$. RTCA Software 2.0 (Roche) was used to calculate the CI values. The CI values were then converted to a percentage $72 \mathrm{~h}$ post-irradiation, with the 0 Gy wells being considered $100 \%$.
Apoptosis detection. A total of $5 \times 10^{4}$ cells were plated onto 24-well tissue culture plates in triplicate for each time point. To detect phosphatidylserine exposure on the outer cell membrane, the cells were irradiated with 0,5 , and $10 \mathrm{~Gy}$ as indicated. Following 24, 48 and $72 \mathrm{~h}$ the supernatant was collected and mixed with the washed, trypsinized cells, prior to incubation with Annexin $\mathrm{V}$ binding buffer containing $0.01 \mathrm{mM}$ HEPES, $0.14 \mathrm{mM} \mathrm{NaCl}$ and $2.5 \mathrm{mM} \mathrm{CaCl}_{2}$. Annexin V-Alexa 488 (2.5:100) (Gibco Life Technologies) and $10 \mu \mathrm{g} / \mathrm{ml}$ propidium iodide (PI) (Sigma-Aldrich Chemie $\mathrm{GmbH}$ ) were added to the cells and incubated for $15 \mathrm{~min}$ in the dark, at room temperature. Following washing, the cells were analyzed using a FACSCalibur cytofluorimeter (BD Biosciences, San Jose, CA, USA). Annexin V-Alexa 488 was used to determine the percentage of positive cells (FLI), and PI was used to determine the percentage of negative early apoptotic cells (FL3).

Statistical analysis. Statistical differences between groups were assessed with a paired Student's t-test, as analyzed using Windows Excel (Microsoft Corporation, Redmond, WA, USA). $\mathrm{P}<0.05$ was considered to indicate a statistically significant difference (Table I). The data are presented as the mean \pm standard deviation.

\section{Results}

The present study evaluated the effects of ionizing radiation on a wide spectrum of malignant human cell lines and human primary fibroblast cells. A systematic comparison of end-point and real-time assays was carried out in order to reveal the advantages of the xCELLigence system.

GBM2 human glioma cells. The colony forming assay served as a reference to assess the effects of radiation on cell growth. A strong correlation was detected between irradiation and colony formation. Significant differences $(\mathrm{P}<0.05)$ were observed between the control cells and those exposed to 5 or 10 Gy irradiation doses. The number of colonies linearly decreased with increasing doses of irradiation (Fig. 1). Four cell densities were tested using the RTCA (Fig. 2A-E), MTS (Fig. 2F), and LDH (Fig. 2G) assays. The effects of irradiation were detected using the label-free method $<24 \mathrm{~h}$ following treatment. At the highest cell density, (4,000 cells/well), the cells were overgrown in both the untreated and irradiated samples $50 \mathrm{~h}$ post-irradiation (Fig. 2D). Cellular exponential growth was arrested due to irradiation at $24 \mathrm{~h}$ which was recorded as a small plateau at 40-60 h in the cells plated at 500 and 1,000 cells/well treated with 5 and 10 Gy irradiation. Following CI curve normalization (the CI values were scaled so that they were independent of initial cell densities) the strongest effects of irradiation were observed at the two lowest cell densities (Fig. 2A and B). Following 5 Gy irradiation, a significant decrease in cellular proliferation was detected, whereas at $10 \mathrm{~Gy}$ irradiation cellular proliferation ceased $60 \mathrm{~h}$ post-irradiation. Upon comparison of the results obtained from the xCELLigence system and the end-point assays, it was apparent that both the LDH and MTS assays (Fig. 2F and G) detected a decrease in the overall enzymatic activity at 500, 1,000 and 2,000 cells/well at $72 \mathrm{~h}$; however, this apparent 


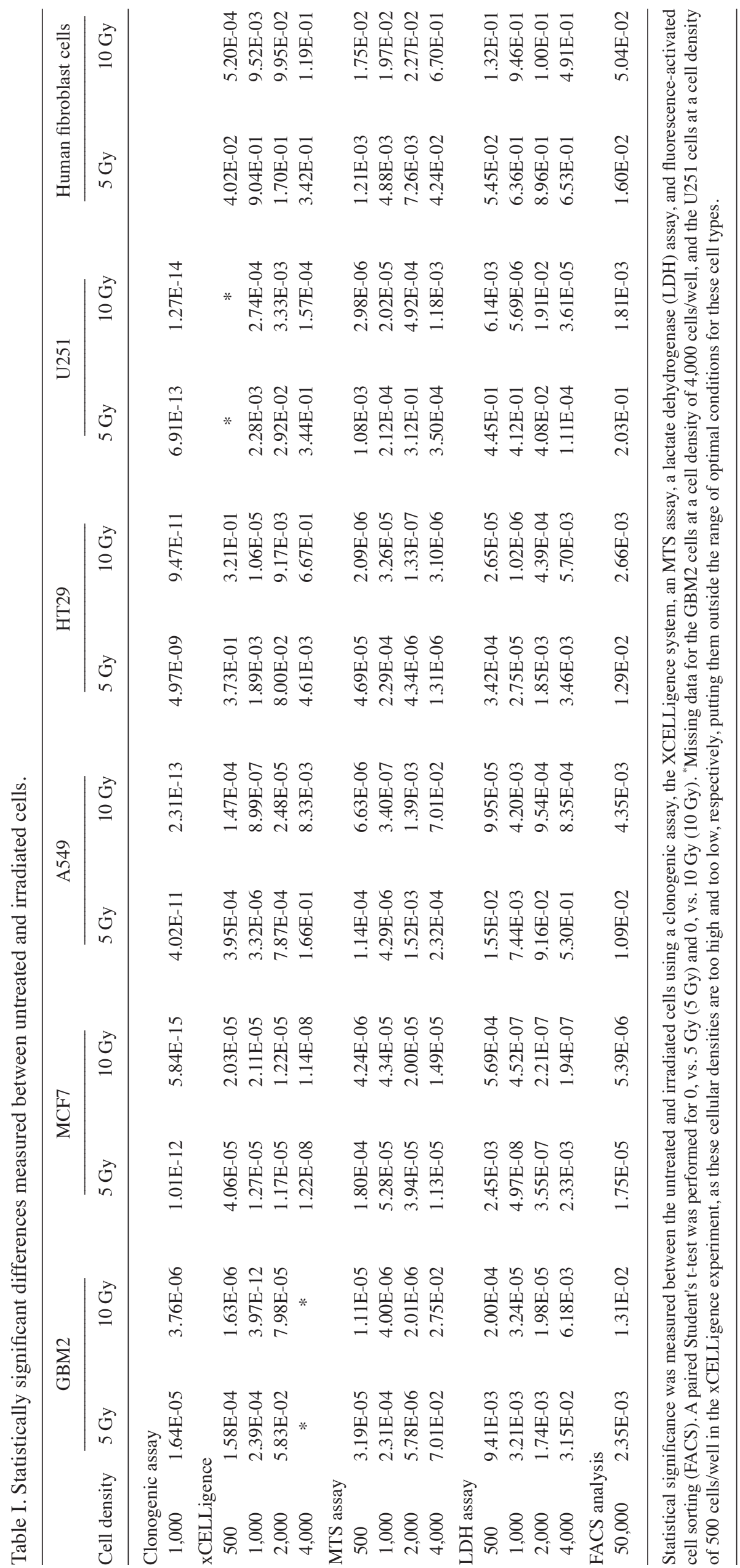


decrease in enzymatic activity was significantly lower, as compared with the impedance measured by the xCELLigence system (Fig. 2E). The end-point assays revealed that the effects of irradiation decreased with the increasing number of seeded cells, except for a density of 4,000 cells/well, as this cell density was too high for impedance to occur. The percentage of early apoptotic cells was detected by Annexin V binding to phosphatidylserine exposed on the outer membrane. The percentage of apoptotic cells was correlated with the dose of irradiation, and following 10 Gy $12 \%$ of cells exhibited early apoptosis (Fig. 2H). Discrepancies in the ratios of all parameters measured by each assay are due to differences in the measured biological targets.

MCF7 human breast adenocarcinoma cells. The results of the colony forming assay revealed significant differences between the 5 and 10 Gy irradiated cells, and the control cells (Fig. 1). In the case of the MTS and LDH assays, all four tested cell densities provided results that correlated with these of the xCELLigence system (Fig. 3). The MCF7 cells were the most radiosensitive of all the experimental cells. Following 5 and $10 \mathrm{~Gy}$ irradiation, the CI values decreased, and after 3 days all $\mathrm{CI}$ values returned to baseline levels suggesting the presence of inhibited cell division and/or marked alterations in cell attachment and shape (Fig. 3A-E). MTS (Fig. 3F) and LDH (Fig. 3G) assays demonstrated a marked decrease in the metabolic activity of the cells at all densities, following $72 \mathrm{~h}$ irradiation. Since the MCF7 cells exhibited high radiosensitivity, $30 \%$ of the MCF7 cells underwent early apoptosis following $72 \mathrm{~h}$ 10 Gy irradiation (Fig. 3H).

A549 human lung carcinoma cells. A strong correlation was detected between irradiation dosage and colony formation. Significant differences $(\mathrm{P}<0.05)$ were observed between the control cells and those exposed to 5 or 10 Gy irradiation (Fig. 1). Using RTCA, MTS and LDH assays, the effects of irradiation could be detected at all four experimental cell densities (Fig. 4). Seeding densities of 500 or 1,000 cells/well proved to be optimal with highly distinguishable growth curves, with the rate of cellular proliferation markedly decreasing following irradiation, in a dose-dependent manner (Fig. 4A, B and E). Seeding densities of 2,000 and 4,000 cells/well resulted in cell overgrowth over the course of 3 days (Fig. 4C, D and E). Although the most pronounced changes were apparent with the xCELLigence system, the effects of irradiation were also shown to decrease with increasing cell density in the MTS (Fig. 4F) and LDH (Fig. 4G) assays. The curves generated following 5 and 10 Gy irradiation were indistinguishable, exhibiting similar apoptotic rates of 22 and $22.8 \%$, respectively (Fig. $4 \mathrm{H}$ ).

HT-29 human colorectal adenocarcinoma cells. In a similar manner to the other cell lines, decreases in HT-29 human colorectal adenocarcinoma cell colony growth were also dose-dependent, exhibiting statistically significant differences between the control and irradiated groups (Fig. 1). All HT-29 cell densities were resistant to all ionizing irradiation doses in terms of cell division and/or changes in morphology, as determined by the xCELLigence system (Fig. 5A-E). Lower cell densities of 1,000 and 2,000 cells/well exposed to a $10 \mathrm{~Gy}$

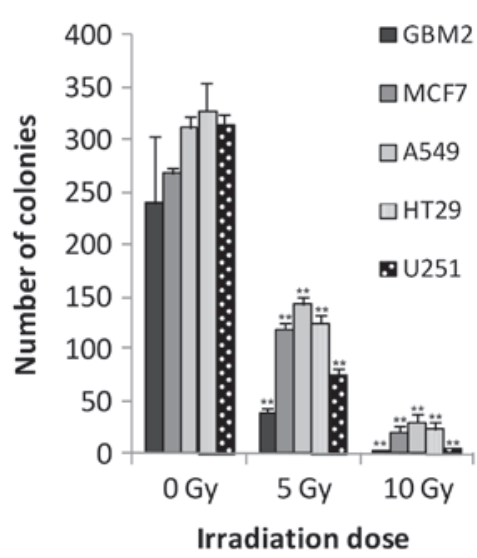

Figure 1. Clonogenic survival of the GBM2, MCF7, A549, HT29, and U251 cells following ionizing radiation. The data are expressed as the mean \pm standard deviation, and all experiments were performed in triplicate. ${ }^{*} \mathrm{P}<0.05$, ${ }^{* *} \mathrm{P}<0.01$ using the paired Student's t-test; treated samples vs. 0 Gy (control) samples.

irradiation (Fig. 5A, B and E) exhibited growth inhibition and/or detachment, results which were concordant with the results from both the MTS and LDH assays. However, in the MTS (Fig. 5F) and LDH (Fig. 5G) assays, all cell densities showed reduced enzymatic activity due to ionizing radiation. As HT-29 cells showed low sensitivity to the 5 and 10 Gy irradiation treatments, only 6 and $10 \%$ of the cells were Annexin V-positive, respectively (Fig. 5H).

U251 human glioblastoma cells. Colony forming capacity was reduced in a dose-dependent manner following irradiation (Fig. 1). A cell density of 500 cells/well did not present sufficient signal throughout the experiment (Fig. 6A), whereas at a cell density of 4,000 cells/well, the cells became overgrown in 2 days in both the control and irradiated samples (Fig. 6D). The results obtained from the RTCA system were significant at cell densities of 1,000 and 2,000 cells/well. At 1,000 cells/well $5 \mathrm{~Gy}$ irradiation dose arrested cell proliferation and/or influenced cell attachment, whereas irradiation with 10 Gy led to a decrease in CI values following $48 \mathrm{~h}$ (Fig. 6B and $\mathrm{E}$ ). A dose-dependent decrease in the CI values was also detectable at a cellular density of 2,000 cells/well, although the decrease was less pronounced than at 1,000 cells/well (Fig. 6C). The MTS and LDH assays showed a significant decrease in the activity levels of dehydrogenases $72 \mathrm{~h}$ after irradiation, although these results were only observable in the LDH assay at a 500 cell/well density (Fig. $6 \mathrm{~F}$ and G). The U251 glioblastoma cell line was moderately sensitive to $\gamma$-irradiation, with an apoptotic cell death of $18 \%$ following 10 Gy irradiation (Fig. 6H).

Human primary fibroblasts. Human primary fibroblasts are unable to form colonies, and were therefore not examined in the colony forming assay. In the RTCA, MTS, and LDH assays, the fibroblasts were more resistant to ionizing radiation, as compared with the experimental malignant cell lines, even following $10 \mathrm{~Gy}$ irradiation, following which cellular proliferation was comparable to that of the control cells (Fig. 7A-G). Similarly to the malignant cell lines, xCELLigence analysis of the fibroblasts showed sensitivity to cell density, with 500 
A

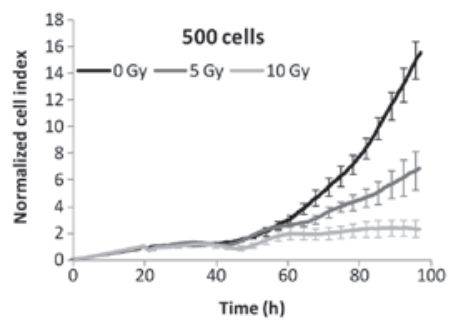

C
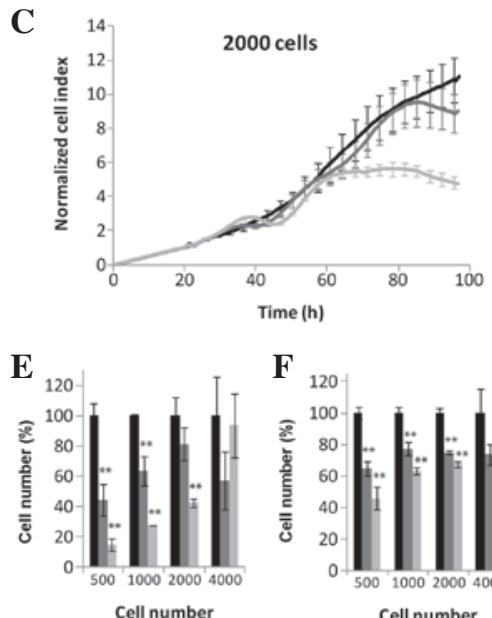

F ${ }_{120}$

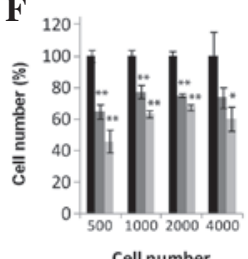

B

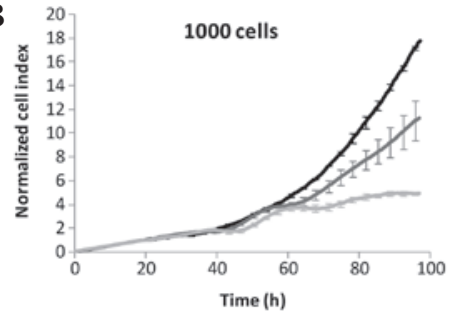

D

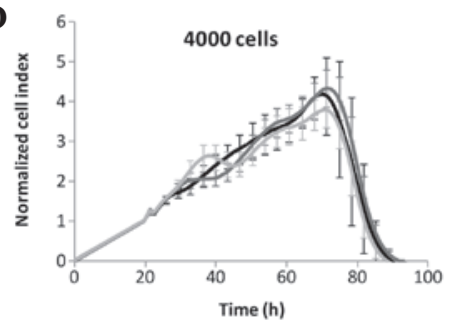

G

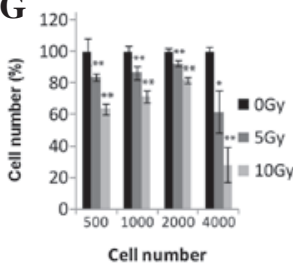

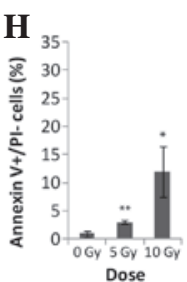

Figure 2. Dynamic monitoring of GBM2 human glioma cell adhesion and proliferation using the xCELLigence system. The GBM2 cells were monitored in 16-well E-plates at densities of (A) 500, (B) 1,000, (C) 2,000, and (D) 4,000 cells/well. (E) Cell index values measured by the xCELLigence system were converted to a percentage $72 \mathrm{~h}$ post-irradiation. Cells treated with an irradiation dose of 0 Gy were considered $100 \%$. An (F) MTS assay and (G) lactate dehydrogenase assay were conducted $72 \mathrm{~h}$ following 5 and $10 \mathrm{~Gy}$ irradiation. (H) Percentage of early apoptotic cells as detected by Annexin V-Alexa 488 and propidium iodide staining, following $72 \mathrm{~h}$ of 5 and $10 \mathrm{~Gy}$ irradiation. The data are expressed as the mean \pm standard deviation. ${ }^{*} \mathrm{P}<0.05$, ${ }^{* *} \mathrm{P}<0.01 \mathrm{using}$ the paired Student's t-test; treated samples vs. 0 Gy (control) samples.

A

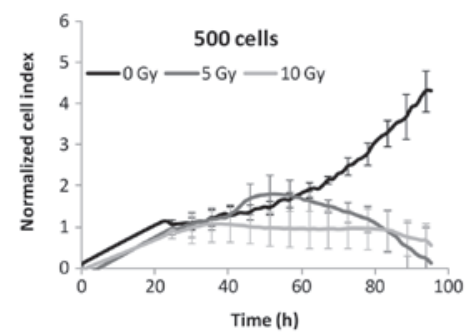

C
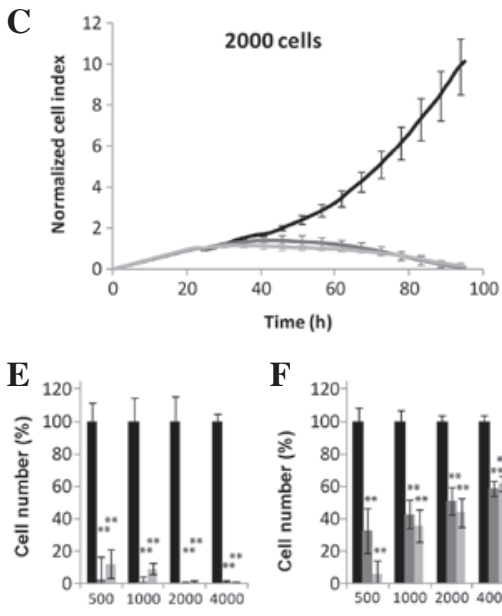

Cell number
$\mathbf{F}_{120}$

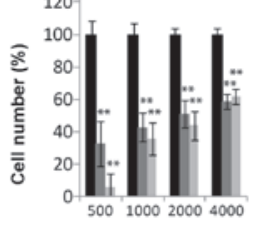

Cell number
B

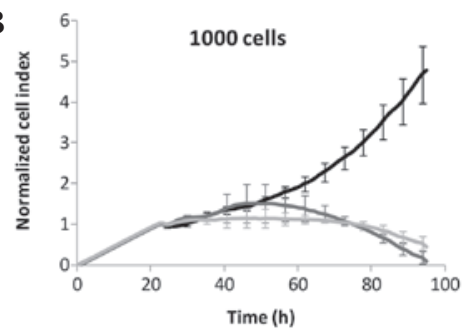

D

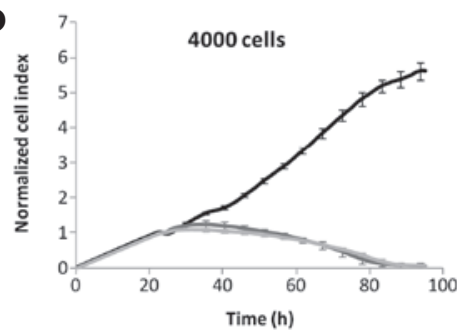

$\mathbf{G}_{120}$
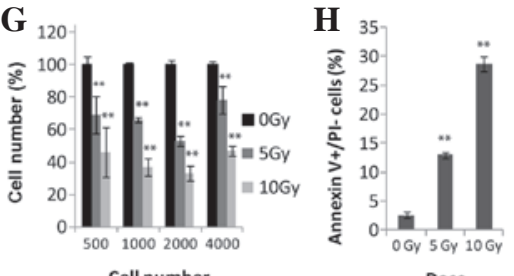

Figure 3. Dynamic monitoring of MCF7 human breast adenocarcinoma cell adhesion and proliferation using the xCELLigence system. The MCF7 cells were monitored in 16-well E-plates at densities of (A) 500, (B) 1,000, (C) 2,000, and (D) 4,000 cells/well. (E) Cell index values measured by the xCELLigence system were converted to a percentage $72 \mathrm{~h}$ post-irradiation. Cells treated with an irradiation dose of 0 Gy were considered $100 \%$. An (F) MTS assay and (G) lactate dehydrogenase assay were conducted $72 \mathrm{~h}$ following 5 and $10 \mathrm{~Gy}$ irradiation. $(\mathrm{H})$ Percentage of early apoptotic cells as detected by Annexin V-Alexa 488 and propidium iodide staining, following $72 \mathrm{~h}$ of 5 and $10 \mathrm{~Gy}$ irradiation. The data are expressed as the mean \pm standard deviation. ${ }^{*} \mathrm{P}<0.05,{ }^{* *} \mathrm{P}<0.01 \mathrm{using}$ the paired Student's t-test; treated samples vs. 0 Gy (control) samples. 
A

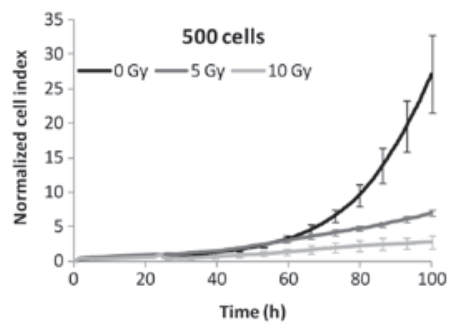

C

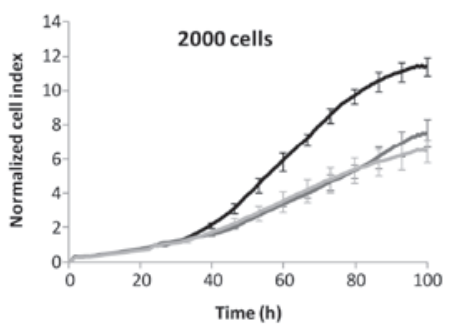

E

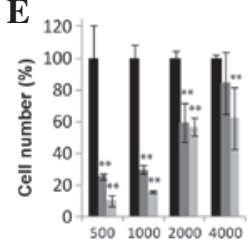

Cell number
$\mathbf{F}_{120}$

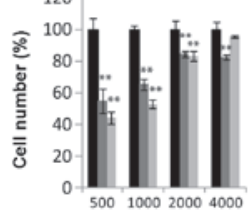

Cell number

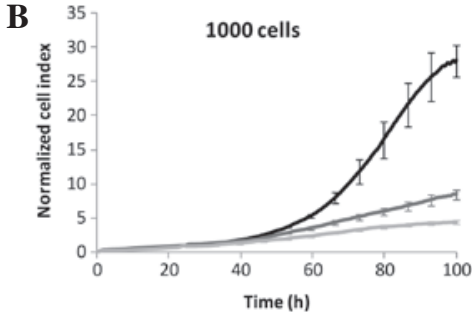

D

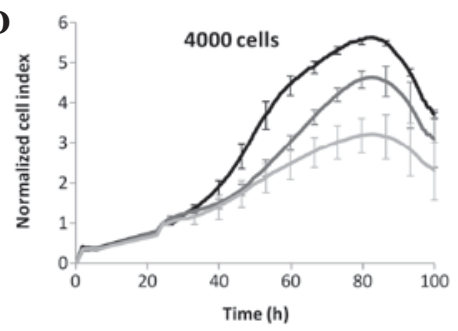

G

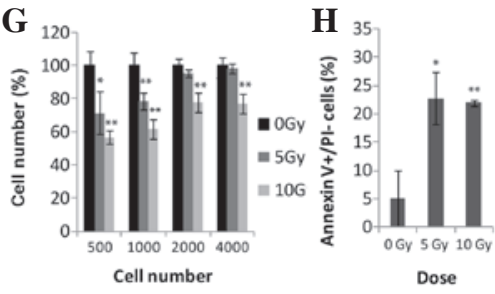

Figure 4. Dynamic monitoring of A549 human lung carcinoma cell adhesion and proliferation using the xCELLigence system. The A549 cells were monitored in 16-well E-plates at densities of (A) 500, (B) 1,000, (C) 2,000, and (D) 4,000 cells/well. (E) Cell index values measured by the xCELLigence system were converted to a percentage $72 \mathrm{~h}$ post-irradiation. Cells treated with an irradiation dose of 0 Gy were considered $100 \%$. An (F) MTS assay and (G) lactate dehydrogenase assay were conducted $72 \mathrm{~h}$ following 5 and $10 \mathrm{~Gy}$ irradiation. (H) Percentage of early apoptotic cells as detected by Annexin V-Alexa 488 and propidium iodide staining, following $72 \mathrm{~h}$ of 5 and $10 \mathrm{~Gy}$ irradiation. The data are expressed as the mean \pm standard deviation. ${ }^{*} \mathrm{P}<0.05,{ }^{* *} \mathrm{P}<0.01$ using the paired Student's t-test; treated samples vs. 0 Gy (control) samples.

A

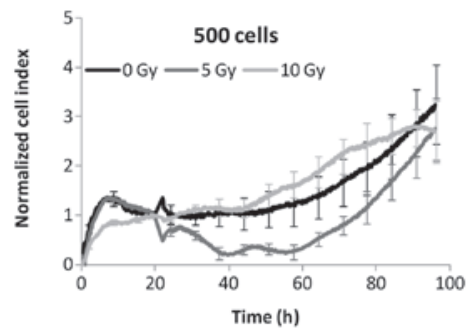

C
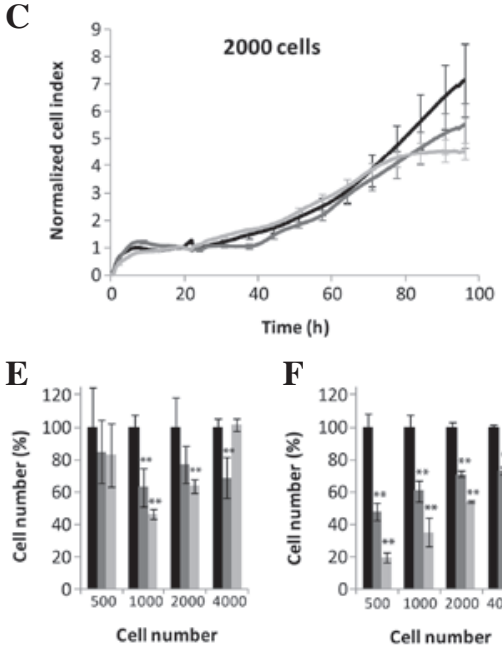

$\mathbf{F}$

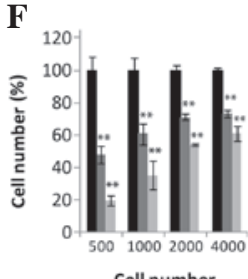

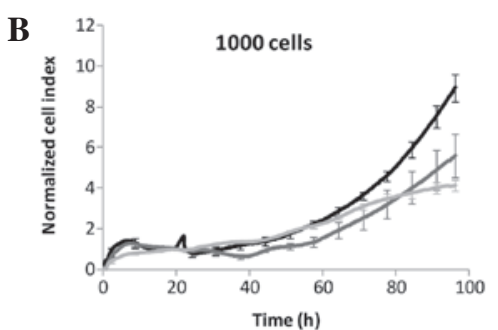

D

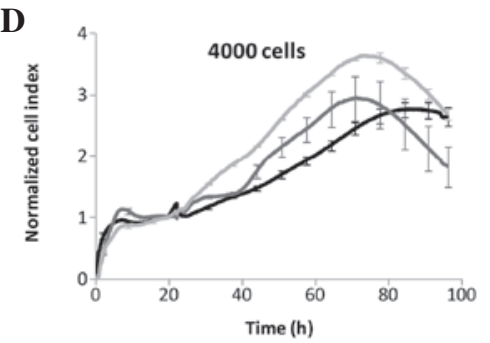

G

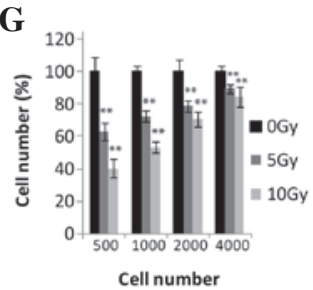

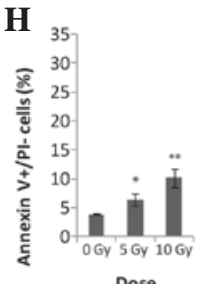

Figure 5. Dynamic monitoring of HT-29 human colorectal adenocarcinoma cell adhesion and proliferation using the xCELLigence system. The HT-29 cells were monitored in 16-well E-plates at densities of (A) 500,(B) 1,000, (C) 2,000, and (D) 4,000 cells/well. (E) Cell index values measured by the xCELLigence system were converted to a percentage $72 \mathrm{~h}$ post-irradiation. Cells treated with an irradiation dose of 0 Gy were considered $100 \%$. An (F) MTS assay and (G) lactate dehydrogenase assay were conducted $72 \mathrm{~h}$ following 5 and $10 \mathrm{~Gy}$ irradiation. (H) Percentage of early apoptotic cells as detected by Annexin V-Alexa 488 and propidium iodide staining, following $72 \mathrm{~h}$ of 5 and $10 \mathrm{~Gy}$ irradiation. The data are expressed as the mean \pm standard deviation. ${ }^{*} \mathrm{P}<0.05,{ }^{* *} \mathrm{P}<0.01$ using the paired Student's t-test; treated samples vs. 0 Gy (control) samples. 
A

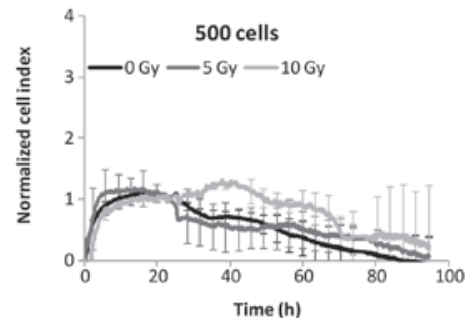

C

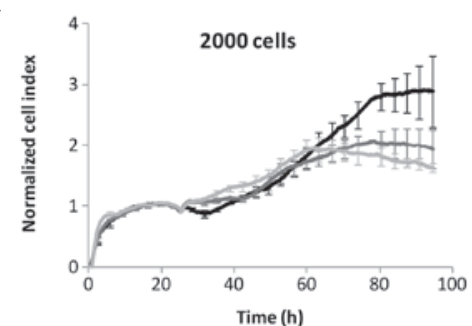

$\mathbf{E}$

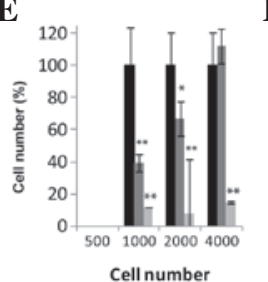

$\mathbf{F}$

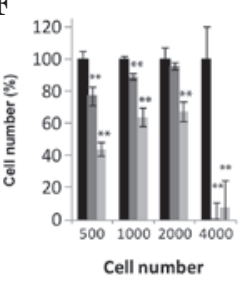

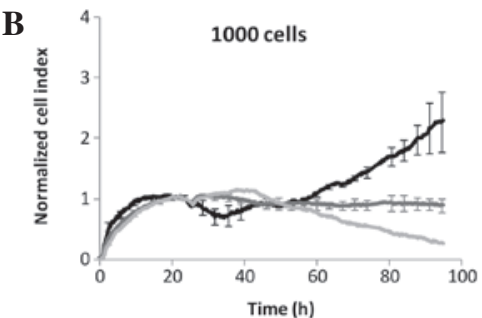

D

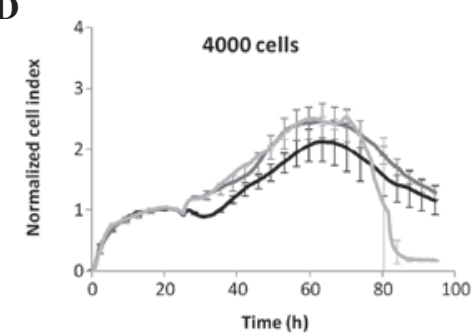

G

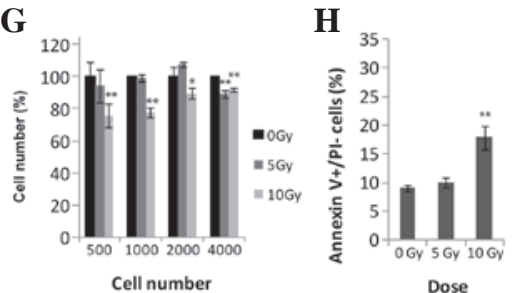

Figure 6. Dynamic monitoring of U251 human glioblastoma cell adhesion and proliferation using the xCELLigence system. The U251 cells were monitored in 16-well E-plates at densities of (A) 500, (B) 1,000, (C) 2,000, and (D) 4,000 cells/well. (E) Cell index values measured by the xCELLigence system were converted to a percentage $72 \mathrm{~h}$ post-irradiation. Cells treated with an irradiation dose of 0 Gy were considered $100 \%$. An (F) MTS assay and (G) lactate dehydrogenase assay were conducted $72 \mathrm{~h}$ following 5 and $10 \mathrm{~Gy}$ irradiation. (H) Percentage of early apoptotic cells as detected by Annexin V-Alexa 488 and propidium iodide staining, following $72 \mathrm{~h}$ of 5 and $10 \mathrm{~Gy}$ irradiation. The data are expressed as the mean \pm standard deviation. ${ }^{*} \mathrm{P}<0.05,{ }^{* *} \mathrm{P}<0.01$ using the paired Student's t-test; treated samples vs. 0 Gy (control) samples.

A

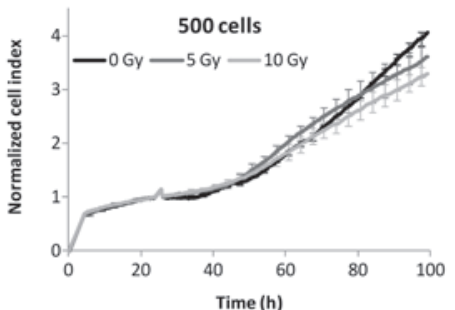

C

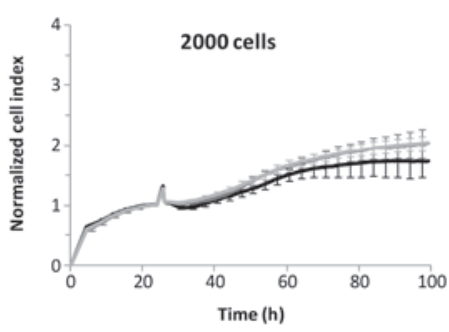

$\mathbf{E}$

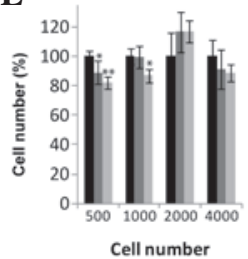

F

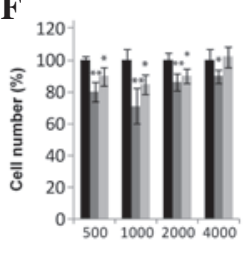

B

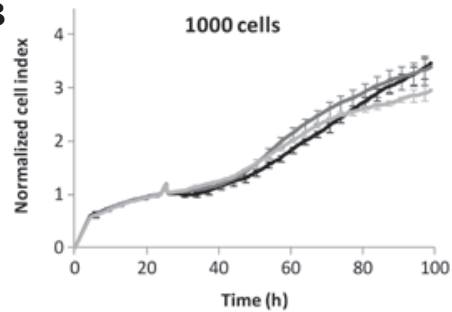

D

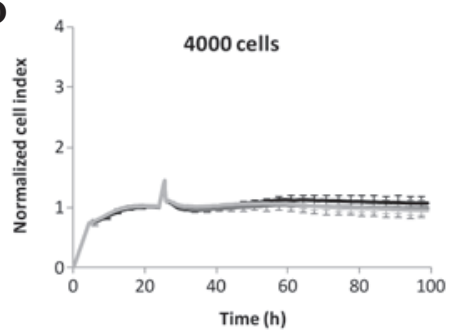

$\mathbf{G}_{1}$
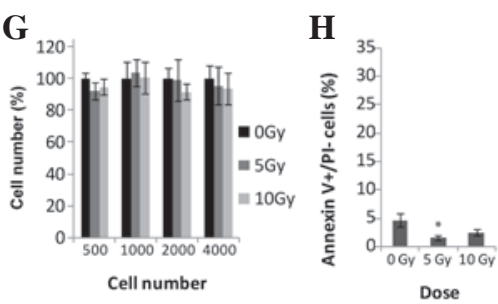

Figure 7. Dynamic monitoring of human primary fibroblast cell adhesion and proliferation using the xCELLigence system. Human primary fibroblast cells were monitored in 16-well E-plates at densities of (A) 500, (B) 1,000, (C) 2,000, and (D) 4,000 cells/well. (E) Cell index values measured by the xCELLigence system were converted to a percentage $72 \mathrm{~h}$ post-irradiation. Cells treated with an irradiation dose of 0 Gy were considered $100 \%$. An (F) MTS assay and (G) lactate dehydrogenase assay were conducted $72 \mathrm{~h}$ following 5 and 10 Gy irradiation. (H) Percentage of early apoptotic cells as detected by Annexin V-Alexa 488 and propidium iodide staining, following $72 \mathrm{~h}$ of 5 and $10 \mathrm{~Gy}$ irradiation. The data are expressed as the mean \pm standard deviation. ${ }^{*} \mathrm{P}<0.05,{ }^{* *} \mathrm{P}<0.01 \mathrm{using}$ the paired Student's t-test; treated samples vs. 0 Gy (control) samples. 
and 1,000 cells/well being the optimal density, whereas higher cellular densities saturated the CI signals prior to the end of the assay (Fig. 7A-D). Concordant with the above-mentioned methods, the human primary fibroblast slowly dividing cells were resistant to both 5 and 10 Gy irradiation doses, with 1.6 and $2.5 \%$ early apoptotic cells, respectively (Fig. $7 \mathrm{H}$ ).

\section{Discussion}

The study of radiotherapy began in 1896 with the discovery of X-rays by Wilhelm Röntgen. Thereafter, radiation became one of the primary strategies for the treatment of numerous malignant tumors, through the induction of DNA damage (22). The use of radiation as a treatment is based on reports of radiation-induced inhibition of cellular proliferation, induction of apoptotic cell death, and inhibition of tumor growth, due to the fact that rapidly dividing malignant cells are more radiosensitive, as compared with normal tissue cellular components (23). Cell survival following radiation is usually measured using end-point assays, and clonogenic and mitochondrial dehydrogenase activity assays, such as MTS, are the most widely-used methods to carry out such research. However, the limitations of end-point assays include the time taken for colonies to form, and the inability to measure proliferation of primary cells, which do not grow as colonies. The MTS assay quantifies metabolically viable cells by measuring their ability to reduce a tetrazolium dye. The present study used numerous additional methods of cell viability detection following irradiation in order to validate the use of the xCELLigence system. Given the low cell densities used in the present study (500, 1,000, 2,000, and 4,000 cells/well), combined with the relatively low rates of cell apoptosis, $\mathrm{LDH}$ release into the supernatant remained under the detection limit, likely due to the low sensitivity of the assay. Therefore, the total LDH detection method was used following cell lysis, which showed good overall correlation with the MTS results. The effects of irradiation were investigated using various methods: The clonogenic method relies on CFU capacity, the xCELLigence system detects impedance influenced by cell attachment, the MTS and LDH assays reflect the metabolic activity levels of viable cells, and Annexin V/PI staining determines cell apoptosis rates.

Compared with conventional end-point cell-based assays, dynamic monitoring of the cell response, such as cell adhesion, spreading, proliferation, and cell death, is one of the advantages of the xCELLigence system. Cell number optimization is usually performed prior to experimentation with a given assay; however, in the present study the impact of cell density on impedance measurements was demonstrated, and 1,000 cells/well was determined to be the optimal cell density on the E-plate 16. At higher cell densities the length of the assay was limited either by the lack of nutrients in the culture media, or by overgrowth of the cells in the well. This overgrowth was represented by a plateau in the CI values. The impedance-based data detected differences in the radiosensitivity between the various types of human malignant and primary cells. As the fastest dividing (data not shown) cells, the MCF7 human breast adenocarcinoma cells were the most sensitive to $\gamma$-rays, corresponding to the lowest metabolic activity levels and the highest rates of apoptosis. The GBM2 human glioblastoma and A549 human lung carcinoma cells also showed high radiosensitivity with dose-dependent diminished proliferation rates, as determined by the impedance measurements obtained with cell densities of 500 and 1,000 cells/well. The differences in responsiveness of the various cells to ionizing radiation with regards to the investigated parameters is a unique characteristic of each cell type. Irradiated GBM2 and A549 cells exhibited high apoptotic rates, as well as low dehydrogenase activity at low cell densities. The U251 human glioblastoma cells were influenced only at 1,000 cells/well in the E-plate. Mitochondrial enzymatic activity significantly diminished, whereas the levels of LDH, a cytoplasmic enzyme, remained unaffected. As radiation induces the intrinsic apoptotic pathway, a substantial portion (17\%) of the cells were apoptotic. HT-29 human colorectal adenocarcinoma cells showed low radiosensitivity irrespective of the irradiation dose according to the E-plate measurements, whereas mitochondrial and cytoplasmic enzyme activity was greatly affected by radiation. As slowly dividing cells, human primary fibroblasts proved to be completely resistant to $\gamma$ photons. Impedance, cellular dehydrogenases and phosphatidylserine exposure remained unaltered. The insensitivity to radiation of the primary fibroblasts may be relevant in clinical settings. Tumor-associated fibroblasts are important cellular components of the stroma of solid tumors, inducing neovascularization, modulating the immune response, and enhancing metastasis (24). Interactions between malignant cells and surrounding stromal cells may have an important role in tumor invasion and metastasis $(25,26)$. A previous study of human retinoblastoma delineated the disadvantages of radiotherapy, where following radiation treatment and tumor regression, fibrovascular proliferation on the retinal surface was observed (27). Radiation of the neoplastic tissues surrounded by stromal components may undesirably affect the progression of cancer, as irradiated fibroblasts enhance the metastatic ability of pancreatic cancer cells (28).

To our knowledge, the present study is the first to demonstrate the application of the xCELLigence system for the determination of the effects of radiation on cells in culture. The results obtained using standard end-point assays correlated with the decline of the CI values detected following irradiation. However, the end-point assays were only able to investigate the reductive capacity of a cell, rather than determining alterations in cell adherence, shape, and size, which are earlier phenotypic changes than cell death itself.

In conclusion, the present study demonstrated that the $\mathrm{xCELLigence} \mathrm{system} \mathrm{is} \mathrm{able} \mathrm{to} \mathrm{detect} \mathrm{cell} \mathrm{responses} \mathrm{to} \mathrm{ionizing}$ radiation, and provides real-time data on the onset of these effects. There are numerous platforms available for the xCELLigence system: Single plate (SP 96, 1x96 wells), double plate (DP 16, 3x16 wells). and multi plate (MP 96, 6x96 wells). The multi plate platform offers the possibility of high-throughput real-time screening of preclinical drugs in vitro, which influence malignant and tumor stromal cell behavior, or examination of the response of biopsy-derived cells to radiation with concomitant medication ex vivo. A low cell density requirement (1,000 cells) is an advantage offered by the xCELLigence system for the study of radiation effects, and being a non-invasive, label-free and real-time method, subsequent analysis, such as determination of DNA or protein expression, or enzyme activity, of the investigated cells would be possible. 


\section{Acknowledgements}

The present study was supported by a grant from "TÁMOP-4.2.1/B-09/1/KONV-2010-0005 Creating the Centre of Excellence at the University of Szeged", supported by the European Union and co-financed by the European Regional Development Fund. The ELI-ALPS project (GOP-1.1.1-12/B-2012-0001) is supported by the European Union and co-financed by the European Regional Development Fund.

\section{References}

1. Bittoni A, Faloppi L, Giampieri R and Cascinu S: Selecting the best treatment for an individual patient. Recent Results Cancer Res 196: 307-318, 2012.

2. Weiss JF and Landauer MR: History and development of radiation-protective agents. Int J Radiat Biol 85: 539-573, 2009.

3. Fietkau R: Concurrent radiochemotherapy for the treatment of solid tumors. Strahlenther Onkol 188 (Suppl 3): 263-271, 2012 (In German).

4. Hendry JH: Radiation biology and radiation protection. Ann ICRP 41: 64-71, 2012.

5. Smout MJ, Kotze AC, McCarthy JS and Loukas A: A novel high throughput assay for anthelmintic drug screening and resistance diagnosis by real-time monitoring of parasite motility. PLoS Negl Trop Dis 16;4: e885, 2010.

6. Buch K, Peters T, Nawroth T, Sänger M, Schmidberger H and Langguth P: Determination of cell survival after irradiation via clonogenic assay versus multiple MTT Assay - a comparative study. Radiat Oncol 7: 1, 2012

7. Price $P$ and McMillan TJ: Use of tetrazolium assay in measuring the response of human tumor cells to ionizing radiation. Cancer Res 50: 1392-1396, 1990.

8. Brubel R, Boronkai A, Reglodi D, Racz B, Nemeth J, Kiss P, Lubics A, Toth G, Horvath G, Varga T, Szogyi D, et al: Changes in the expression of pituitary adenylate cyclase-activating polypeptide in the human placenta during pregnancy and its effects on the survival of JAR choriocarcinoma cells. J Mol Neurosci 42: 450-458, 2010.

9. Caltová K and Cervinka M: Antiproliferative effects of selected chemoterapeutics in human ovarian cancer cell line A2780. Acta Medica (Hradec Kralove) 55: 116-124, 2012.

10. Mishra J, Mittra B and Mittra A: Effect of whole body gamma radiation on hepatic LDH activity, lactate, pyruvate concentration and rate of oxygen consumption in Bufo melanostictus. Indian J Exp Biol 40: 1310-1313, 2002.

11. Ozsvári B,Puskás LG, Nagy LI, Kanizsai I, Gyuris M, Madácsi R, Fehér LZ, Gerö D and Szabó C: A cell-microelectronic sensing technique for the screening of cytoprotective compounds. Int J Mol Med 25: 525-530, 2010.

12. Kiss L, Walter FR, Bocsik A, Veszelka S, Ozsvári B, Puskás LG, Szabó-Révész P and Deli MA: Kinetic analysis of the toxicity of pharmaceutical excipients Cremophor EL and RH40 on endothelial and epithelial cells. J Pharm Sci 102: 1173-1181, 2013.
13. Kürti L, Veszelka S, Bocsik A, Dung NT, Ozsvári B, Puskás LG, Kittel A, Szabó-Révész P and Deli MA: The effect of sucrose esters on a culture model of the nasal barrier. Toxicol In Vitro 26: 445-454, 2012

14. Kürti L, Veszelka S, Bocsik A, Ozsvári B, Puskás LG, Kittel A, Szabó-Révész P and Deli MA: Retinoic acid and hydrocortisone strengthen the barrier function of human RPMI 2650 cells, a model for nasal epithelial permeability. Cytotechnology 65: 395-406, 2013.

15. Atienza JM, Yu N, Kirstein SL, Xi B, Wang X, Xu X and Abassi YA: Dynamic and label-free cell-based assays using the real-time cell electronic sensing system. Assay Drug Dev Technol 4: 597-607, 2006.

16. Urcan E, Haertel U, Styllou M, Hickel R, Scherthan H and Reichl FX: Real-time xCELLigence impedance analysis of the cytotoxicity of dental composite components on human gingival fibroblasts. Dent Mater 26: 51-58, 2010.

17. Ke N, Wang X, Xu X and Abassi YA: The xCELLigence system for real-time and label-free monitoring of cell viability. Methods Mol Biol 740: 33-43, 2011.

18. Diemert S, Dolga AM, Tobaben S, Grohm J, Pfeifer S, Oexler E and Culmsee C: Impedance measurement for real-time detection of neuronal cell death. J Neurosci Methods 203: 69-77, 2012.

19. Erskine CL, Henle AM and Knutson KL: Determining optimal cytotoxic activity of human Her2neu specific CD8 T cells by comparing the Cr51 release assay to the xCELLigence system. J Vis Exp (66): e3683, 2012.

20. Shareef MM, Brown B, Shajahan S, Sathishkumar S, Arnold SM, Mohiuddin M, Ahmed MM and Spring PM: Lack of P-glycoprotein expression by low-dose fractionated radiation results from loss of nuclear factor-kappaB and NF-Y activation in oral carcinoma cells. Mol Canc Res 6: 89-98, 2008.

21. Schwarz SB, Schaffer PM, Kulka U, Ertl-Wagner B, Hell R and Schaffer M: The effect of radio-adaptive doses on HT-29 and GM637 cells. Radiat Oncol 3: 12, 2008

22. Feofanova N, Geraldo JM and de Andrade LM: Radiation oncology in vitro: Trends to improve radiotherapy through molecular targets. Biomed Res Int 2014: 461687, 2014.

23. Hendry JH and West CM: Apoptosis and mitotic cell death: Their relative contributions to normal-tissue and tumour radiation response. Int J Radiat Biol 71: 709-719, 1997.

24. Togo S, Polanska UM, Horimoto $Y$ and Orimo A: Carcinoma-associated fibroblasts are a promising therapeutic target. Cancers (Basel) 5: 149-169, 2013.

25. Grey AM, Schor AM, Rushton G, Ellis I and Schor SL: Purification of the migration stimulating factor produced by fetal and breast cancer patient fibroblasts. Proc Natl Acad Sci USA 86: 2438-2442, 1989.

26. Camps JL, Chang SM, Hsu TC, Freeman MR, Hong SJ, Zhau HE, von Eschenbach AC and Chung LW: Fibroblast-mediated acceleration of human epithelial tumor growth in vivo. Proc Natl Acad Sci USA 87: 75-79, 1990.

27. Albert DM, Walton DS, Weichselbaum RR, Cassady JR, Little JB, Leombruno D, Trantravahi R and Puliafito CA: Fibroblast radiosensitivity and intraocular fibrovascular proliferation following radiotherapy for bilateral retinoblastoma. $\mathrm{Br}$ J Ophthalmol 70: 336-342, 1986.

28. Ohuchida K, Mizumoto K, Murakami M, Qian LW, Sato N, Nagai E, Matsumoto K, Nakamura T and Tanaka M: Radiation to stromal fibroblasts increases invasiveness of pancreatic cancer cells through tumor-stromal interactions. Cancer Res 64: 3215-3222, 2004. 